\title{
Cerebrospinal fluid tau protein as a biochemical marker for Alzheimer's disease: a community based follow up study
} N Andreasen, E Vanmechelen, A Van de Voorde, P Davidsson, C Hesse, S Tarvonen,
I Räihä, L Sourander, B Winblad, K Blennow

\section{Abstract}

Objectives-Biochemical markers for Alzheimer's disease would be of great value, especially to help in diagnosis early in the course of the disease. A pronounced increase in CSF tau protein (CSF-tau) is found in most patients with Alzheimer's disease. However, the specificity has to be further studied, as an increase in CSF-tau has also been found in other dementias, especially in vascular dementia. As most previous CSF studies have been based on selected inpatients, it was considered of special interest to examine the diagnostic potential of CSF-tau in a community population based sample of consecutive patients with dementia. Such patient material has been examined at the Piteå River Valley Hospital in Northern Sweden since 1986, and includes all those with memory disturbances in the community. The aim was also to study if an increase in CSF-tau is found early in the disease process, and whether CSF-tau changes during the progression of disease.

Methods-Participants: Community population based sample of 75 demented patients (43 with Alzheimer's disease, 21 with vascular dementia, and 11 with mixed Alzheimer's disease/vascular dementia), 18 healthy subjects, and 18 neurological controls. A follow up investigation (including analysis of a new CSF sample) was performed in all patients after about one year.

Main outcome measures-Concentrations of total (both normal tau and PHF-tau) tau in CSF, clinical measures (duration and severity of dementia), and apoE polymorphism.

Results-CSF-tau was markedly increased in Alzheimer's disease, 41/43 $(95 \%)$ patients had values above the cut off level (mean+2 SD) in controls $(306 \mathrm{pg} / \mathrm{ml})$. High CSF-tau concentrations were also found in most patients with vascular dementia, preferentially in patients with vascular dementia without progressive leukoaraiosis on CT, whereas patients with vascular dementia with progressive leukoaraiosis had normal CSF-tau. Concentrations of CSF-tau were stable at one year follow up in both patients with Alzheimer's disease and patients with vascular dementia, and there was no correlation between CSF-tau and either duration or severity of dementia.

Conclusions-The findings confirm the high sensitivity of CSF-tau for the diagnosis of Alzheimer's disease, but high CSFtau was also found in vascular dementia, resulting in a lower specificity. However, high CSF-tau is preferentially found in patients with vascular dementia without progressive leukoaraiosis, which may constitute a group with concomitant Alzheimer's disease pathology. High CSF-tau may be present during the whole course of the disease in Alzheimer's disease. Possibly, therefore, the same high CSF-tau concentrations may be present before the onset of clinical dementia. Follow up studies on such patients will tell whether analysis of CSF-tau is useful as a biochemical marker for early Alzheimer's disease.

\section{(F Neurol Neurosurg Psychiatry 1998;64:298-305)}

Keywords: Alzheimer's disease; biochemical markers; cerebrospinal fluid; tau protein

Alzheimer's disease is the most common form of dementia. Although familial forms of Alzheimer's disease exist, most patients have no clear family history, and are classified as having sporadic Alzheimer's disease. Today, the diagnosis of sporadic Alzheimer's disease is based on clinical exclusion criteria, ${ }^{1}$ and diagnosis is only definite at necropsy. Therefore, both in clinical routine and in view of emerging therapeutic compounds, there is a great need for biochemical diagnostic markers of Alzheimer's disease, especially to help in the diagnosis early in the course of the disease. Moreover, biochemical markers might also be useful to monitor the effect of new potential therapeutic compounds during treatment trials.

As the intercellular space in the brain is in direct contact with the CSF, biochemical changes in the brain may be reflected by CSF analyses. Besides an increased number of senile plaques and neurofibrillary tangles, the central neuropathological findings in Alzheimer's disease consist of a degeneration of the neurons and their synapses. ${ }^{2}{ }^{3}$ Thus analysis of neuronal and synaptic proteins in CSF may function as biochemical markers for the neuronal degeneration in Alzheimer's disease.

One such neuronal protein is tau protein, a normal human brain phosphoprotein, which 


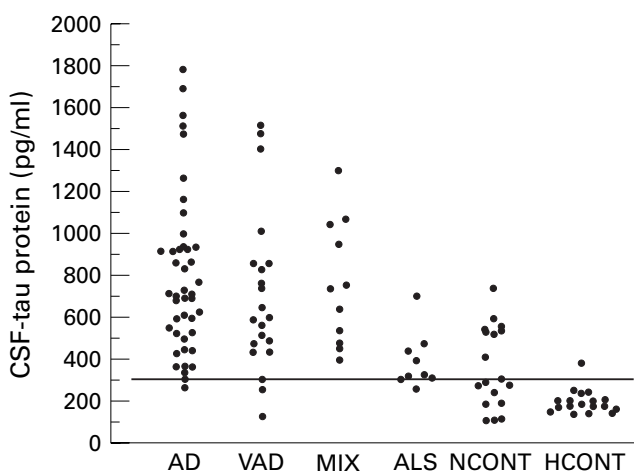

Figure 1 Individual values for CSF-tau in a community based patient sample. AD=Alzheimer's disease, VAD =vascular dementia, $M I X=$ mixed Alzheimer and vascular dementia, $A L S=$ amyotrophic lateral sclerosis. The line represents the cut off level (mean (2SD)) in healthy controls (304 pg/ml). Alzheimer's disease v ALS: $p<0.002$; Alzheimer's disease $v$ neurological controls: $p<0.0001$; Alzheimer's disease $v$ healthy controls: $p<0.0001$; vascular dementia $v$ neurological controls: $p<0.005$; vascular dementia $v$ healthy controls: $p<0.0001 ; M I X v$ neurological controls: $p<0.005 ;$ MIX $v$ healthy controls: $p<0.0001$.

binds to microtubules in the neuronal axons, thereby promoting microtubule assembly and stability. ${ }^{4}$ Several previous studies have found an increase in CSF tau protein (CSF-tau) in Alzheimer's disease.$^{5-19}$ However, the specificity of CSF-tau has not been well established, as an increase in CSF-tau has also been found in some studies in vascular dementia, ${ }^{73}$ although not in all. ${ }^{11}$ Moreover, other than a epidemiological study on very old (85 years of age) people, ${ }^{13}$ most studies have been based on selected hospital patients. In the Piteå River Valley, all those with memory disturbances must be admitted for medical examination to the Piteå River Valley Hospital. Such a patient sample provides a unique opportunity to study the diagnostic potential of a biochemical marker for Alzheimer's disease. Therefore, we set out to examine CSF-tau in this community based patient sample.

Although an increase in CSF-tau has been confirmed in several cross sectional studies, none has examined longitudinal CSF samples from individual patients. Thus our aim was also to examine if CSF-tau concentration changes over time during the disease process, by analysing paired samples at baseline and one year follow up, and by studying the relation between CSF-tau and duration of disease. Our interest was to see if high CSF-tau concentrations are present early in the disease process, and consequently whether CSF-tau may be of use as a biochemical marker early in the disease in patients with Alzheimer's disease. Similarly, it is still an open question whether CSF-tau increases with increasing severity of dementia. Therefore, we also studied the relation between CSF-tau and severity of dementia.

Finally, apolipoprotein E (apoE) has been found to be involved in the pathogenesis of Alzheimer's disease. Higher frequency of the ApoE4 allele is found in patients with Alzheimer's disease than in the general population. ${ }^{20-23}$ However, the pathogenetic mechanism of ApoE4 in Alzheimer's disease is unknown. In vitro studies have shown that ApoE3 binds to the microtubule associated protein tau with high avidity, whereas ApoE4 does not bind tau, suggesting that ApoE3, but not ApoE4, by binding to tau, slows the degree of tau phosphorylation and self assembly into paired helical filaments. ${ }^{24}$ It has also been suggested that intraneuronal ApoE, by interaction with tau protein, may influence the neuronal pathology in Alzheimer's disease, from early in the disease. ${ }^{25}$ Therefore, we also found it of interest to study if CSF-tau differs between patients with Alzheimer's disease with and without the ApoE4 allele.

\section{Materials and methods}

STUDY POPULATION

The investigation was part of the longitudinal geriatric population study in Piteå, in the northern part of Sweden (Andreasen et al, unpublished data), which started in January 1986. Piteå River Valley Hospital is run by the County health authority of Norrbotten. The hospital is the only one in the area, and serves the Piteå River Valley population of about 60 000. The four communities in Piteå River Valley have a common policy, that all of those with memory disturbance must be provided an admission for a medical examination at the Department of Rehabilitation at the hospital. For the present study, we included all patients admitted to the hospital during a six year period, where it was possible to perform a comprehensive one year follow up examination, including a lumbar puncture, in 75 out of 377 patients.

The population based patient sample included 43 patients with Alzheimer's disease (mean age (SD) 75 (7) years), 21 with vascular dementia (79 (5) years), and 11 with mixed Alzheimer's disease/vascular dementia(MIX) (79 (7) years). A follow up investigation (including a new CSF sample) was performed in all patients after about one year.

The clinical evaluation of the patients was performed in a standardised way, and all data were recorded in research protocols at the time of investigation. All patients underwent a comprehensive clinical examination including medical history, physical, neurological, and psychiatric examination, routine laboratory tests, EEG, and brain CT. All examinations were performed or supervised by one of us (NA). In the final diagnostic assessment information from all these tests was used. The presence and severity of dementia was diagnosed according to the DSM-11l-R criteria. ${ }^{26}$ Patients with dementia were classified into diagnostic subgroups: probable Alzheimer's disease according to the NINCDS-criteria ${ }^{1}$; probable vascular dementia, and MIX according to the NINDS-AIREN criteria. $^{27}$ All clinical diagnoses were set individually by two of the authors (NA and $\mathrm{KB}$ ).

To judge the severity of the dementia we used the mini mental state examination (MMSE), ${ }^{28}$ which was performed by a nurse specially trained to adhere to a standardised protocol. The mean MMSE score did not significantly differ between the Alzheimer's disease (20 (6.5), vascular dementia (23 (5.8), 


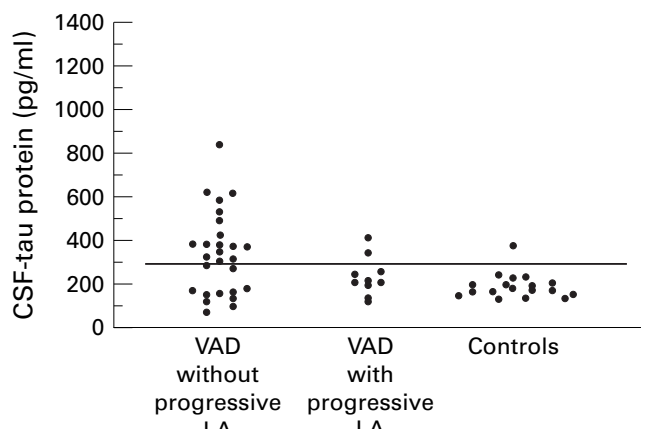

Figure 2 CSF-tau protein in patients with vascular dementia with and without progressive leukoaraiosis. $V A D=$ vascular dementia; $L A=$ leukoaraiosis. The line represents the cut off level (mean (2SD)) in healthy controls (304 pg/ml). Total VAD v controls: $p<0.01$; VAD without progressive LA v controls: $p<0.005 ;$ VAD with progressive LA v controls: NS.

and MIX (21 (5.9) groups. Nor did the duration of dementia significantly differ between the Alzheimer's disease (45 (27) months), vascular dementia (39 (21) months), and MIX (33 (21) months) groups.

As a contrast group, we studied nine patients with amyotrophic lateral sclerosis (age 72 (6) years), diagnosed according to the ICD-9 criteria, from the Department of Neurology, Boden Hospital, Sweden.

We also studied another vascular dementia group, consisting of 37 patients (age 74 (10) years) from the Department of Geriatrics, University of Turku, Finland. This group was studied as a follow up investigation, also including CT, was performed after about three years, to evaluate progression of leukoaraiosis. Leukoaraiosis was defined as confluent areas with reduced attenuation around the lateral ventricles, and the progression of leukoaraiosis was evaluated by comparing the extent of leukoaraiosis at baseline with three year follow up investigations. ${ }^{29}$

As control groups, we studied two different sets of patients. The "neurological" control (NCONT) group came from the Department. of Neurology, Boden Hospital, Sweden, and consisted of 18 patients (age 64 (11) years) with mild neurological symptoms such as headache and vertigo, in which the medical examination did not disclose any organic brain disorder. None of the patients had cognitive symptoms, and all patients were also followed up through medical records for 10 years and none developed dementia. The healthy control group (HCONT), from the Department of Psychiatry, Mölndal Hospital, Sweden, ${ }^{7}$ consisted of 18 subjects (age 67 (4) years), without histories, symptoms, or signs of psychiatric or neurological disease, malignant disease, or systemic disorders (for example, rheumatoid arthritis, infectious disease). No subject had cognitive symptoms and for those over 60 years of age the cognitive status was examined using the MMSE. ${ }^{28}$ Those with scores below 28 were not included.

The mean age was significantly lower in the NCONT group than in the vascular dementia $(p<0.0001)$, MIX $(p<0.0001)$, and Alzheimer's disease $(p<0.0001)$ groups. The mean age

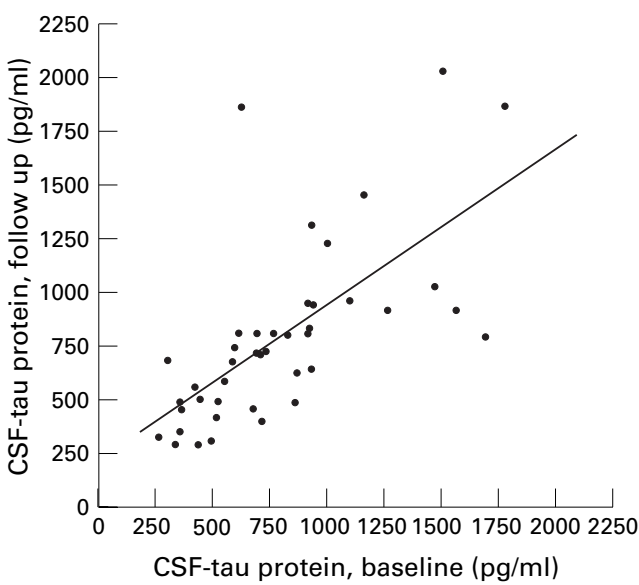

Figure 3 CSF-tau protein at baseline and follow up in Alzheimer's disease. The line represents the regression line for the correlation. Spearman's $r=0.77 ; p<00001$.

was also significantly lower in the healthy control group than in the vascular dementia $(p<0.0001)$, MIX $(p<0.001)$, and Alzheimer's disease $(\mathrm{p}<0.05)$ groups.

All clinical diagnoses and evaluations were made without knowledge of the results from the biochemical analyses and vice versa. The study was approved by the ethics committees at the Universities of Umeå, Göteborg, and Turku. The ethical approval included longitudinal examinations and lumbar punctures.

ANALYSES OF CSF

Samples of CSF were taken between 1100$1200 \mathrm{am}$, in the L3/L4 interspace. The first 12 $\mathrm{ml}$ portion of CSF was collected in polystyrene tubes. After centrifugation to eliminate cells and other insoluble material, all CSF samples were frozen at $-70^{\circ} \mathrm{C}$ pending biochemical analyses. CSF-tau was determined using a sandwich ELISA (Innotest hTAU-Ag, Innogenetics, Ghent, Belgium), constructed to measure total tau (both normal tau and PHF-tau), as described previously in detail..$^{7}$

DETERMINATION OF APOLIPOPROTEIN E ISOFORMS Determination of the ApoE isoforms was performed using isoelectric focusing and western blotting, with minor modifications. ${ }^{30}$ Briefly, serum samples were incubated with neuraminidase to remove sialic acids. Lipoproteins were isolated by precipitation with tungstophosphoric acid and $\mathrm{MgCl}_{2}$. Lipids were extracted using ethanol/diethylether. The remaining apolipoproteins were dissolved in Tris/dithiothreitol/urea buffer, separated by isoelectric focusing, blotted on to a nitrocellulose membrane, and detected using a specific mouse monoclonal antibody against ApoE (Boehringer Mannheim). Serum was available for apoE phenotyping from 35/43 Alzheimer's disease, 21/21 vascular dementia, and 10/11 MIX patients from the population based sample.

STATISTICAL ANALYSIS

In all diagnostic groups, CSF-tau followed the normal distribution curve, but with a positive skewness, and the variance was also higher in 
Table 1 Sensitivity and specificity of CSF-tau in a population based sample

\begin{tabular}{|c|c|c|c|c|c|c|}
\hline Group & No & $\begin{array}{l}\text { CSF-tau (pg/ml) } \\
(\text { mean }(S D))\end{array}$ & Increase (\%) & $\begin{array}{l}\text { Sensitivity } \\
(\%)\end{array}$ & $\begin{array}{l}\text { Specificity } \\
(\%)\end{array}$ & $\begin{array}{l}\text { Positive predictive value } \\
(\%)\end{array}$ \\
\hline $\mathrm{AD}$ & 43 & $796(382)$ & 419 & 95 & & 48 \\
\hline MIX & 11 & $756(296)$ & 398 & 100 & & \\
\hline VAD & 21 & $708(382)$ & 373 & & 14 & \\
\hline ALS & 9 & $386(135)$ & 203 & & 33 & \\
\hline Neurological controls & 18 & $356(193)$ & 187 & & 56 & \\
\hline Healthy controls & 18 & $190(57)$ & & & 94 & \\
\hline
\end{tabular}

$\mathrm{CSF}=$ cerebrospinal fluid; $\mathrm{AD}=$ Alzheimer's disease; $\mathrm{MIX}=$ mixed Alzheimer and vascular dementia; VAD = vascular dementia; $\mathrm{ALS}=$ amyotrophic lateral sclerosis; sensitivity = proportion of $\mathrm{AD}$ cases with high CSF-tau concentrations; specificity $=$ proportion of patients with other diagnoses with normal CSF-tau concentrations; positive predictive value = proportion of correctly diagnosed AD patients of all studied (diseased) patients.

groups with higher means. Therefore, all data were $\log$ transformed before statistical comparisons between groups, which was performed using factor analysis of variance (ANOVA), with post hoc analyses (Tukey's HSD test for unequal n) for comparisons between two groups. The cut off level (mean $+2 \mathrm{SD}$ ) for CSF-tau in the HCONT group was also determined after log transformation. The Spearman's correlation coefficient $(r)$ was used for correlations.

\section{Results}

There were no significant correlations between age and CSF-tau, either in the Alzheimer's disease $(r=-0.03)$, vascular dementia $(r=-0.01)$, MIX $(r=-0.01)$, NCONT $(r=0.41)$, or HCONT $(r=0.13)$ groups.

At baseline investigation, CSF-tau was increased in Alzheimer's disease (796 (382) $\mathrm{pg} / \mathrm{ml}$ ) compared with amyotrophic lateral sclerosis (386 (135) $\mathrm{pg} / \mathrm{ml} ; \mathrm{p}<0.002)$, NCONT (356 (193) $\mathrm{pg} / \mathrm{ml} ; \mathrm{p}<0.0001$ ), and HCONT (190 (57) $\mathrm{pg} / \mathrm{ml} ; \mathrm{p}<0.0001$ ), whereas it did not significantly differ between the Alzheimer's disease and vascular dementia (708 (382) $\mathrm{pg} / \mathrm{ml}$ ) or MIX (756 (296) pg/ml) groups. There was also a significant increase in CSF-tau in vascular dementia compared with NCONT $(\mathrm{p}<0.005)$ and HCONT $(\mathrm{p}<0.0001)$, and in MIX compared with NCONT $(\mathrm{p}<0.005)$ and HCONT $(p<0.0001)$. Figure 1 gives the individual values for CSF-tau protein at baseline.

Using $306 \mathrm{pg} / \mathrm{ml}$ as a cut off value, we calculated the sensitivity and specificity of CSF-tau as a diagnostic marker for Alzheimer's disease. The sensitivity was defined as the ability of CSF-tau to identify Alzheimer's disease - that is, the proportion of patients with Alzheimer's disease with high CSF-tau concentrations. The specificity was defined as the ability of CSF-tau to exclude Alzheimer's disease-that is, the proportion of patients with other diagnoses with normal CSF-tau concentrationswhereas the positive predictive value was defined as the proportion of correctly diagnosed patients with Alzheimer's disease of all patients with high CSF-tau. ${ }^{31}$

CSF-tau was also increased in vascular dementia samples from Turku, Finland (310 (173) $\mathrm{pg} / \mathrm{ml}$ ) compared with HCONT (190 (57); $\mathrm{p}<0.01)$. However, when the vascular dementia group was divided into patients without $(n=27)$ and with $(n=10)$ progressive leukoaraiosis at one year follow up, an increase in CSF-tau was only found in vascular dementia without progressive leukoaraiosis (338
(189) $\mathrm{pg} / \mathrm{ml} ; \mathrm{p}<0.005)$, but not in vascular dementia with progressive leukoaraiosis (234 (89) $\mathrm{pg} / \mathrm{ml}$ ) compared with HCONT (fig 2).

We then determined whether CSF-tau changed during the course of disease. CSF-tau did not significantly differ between baseline and follow up investigations, neither in the Alzheimer's disease group (796 (382) v 791 (411) $\mathrm{pg} / \mathrm{ml}$ ), the vascular dementia group (708 (382) v $695(378) \mathrm{pg} / \mathrm{ml})$, or the MIX group (756 (296) v $697(287) \mathrm{pg} / \mathrm{ml})$. Within the Alzheimer's disease group, there was a significant correlation $(r=0.77 ; \mathrm{p}<00001)$ between baseline and follow up CSF-tau concentrations (fig 3). Similar correlations were found within the vascular dementia $(r=0.91$; $\mathrm{p}<0.001)$ and MIX $(r=0.72 ; \mathrm{p}<0.02)$ groups. There was no significant correlation between duration of dementia and CSF-tau either in the Alzheimer's disease group $(r=0.22)$, or in the vascular dementia $(r=-0.19)$ or the MIX group $(r=0.07)$. There was no significant correlation between severity of dementia, estimated using MMSE, and CSF-tau, either in the Alzheimer's disease group $(r=-0.15)$, or in the vascular dementia $(r=0.17)$ or the MIX group $(r=-0.26)$.

We then studied the rate of progression of dementia in Alzheimer's disease, measured as the change in MMSE between baseline and follow up investigations, and CSF-tau. There were no significant correlations between rate of progression of dementia and CSF-tau $(r=-0.04)$, nor was there any significant correlation between rate of progression of dementia and change in CSF-tau between baseline and follow up ( $r=0.03)$.

Within the Alzheimer's disease group, there were no significant differences between patients without and with the ApoE4 allele, for age $(78(7.4)$ v 75 (6.4) years), duration of dementia (42 (27) v 49 (30) months), or severity of dementia (MMSE score 18 (5.7) v 20 (6.9)). Similarly, within the vascular dementia group, there were no significant differences between patients without and with the ApoE4 allele, for age $(78(4.5)$ v 79 (5.1) years), duration of dementia (37 (28) v 40 (17) months), or severity of dementia (MMSE score $24(5.2) \mathrm{v}$ 22 (6.2)). When comparing CSF-tau concentrations between patients with and without the ApoE4 allele, no significant differences were found, either within the Alzheimer's disease group ( $\mathrm{n}=13$ without ApoE4; CSF-tau 738 (323) $\mathrm{pg} / \mathrm{ml}$ v n=22 with ApoE4; CSF-tau 875 (415) $\mathrm{pg} / \mathrm{ml}$ ), or the vascular dementia group ( $n=8$ without ApoE4; CSF-tau 765 (505) 
Table 2 CSF-tau protein: sensitivity and specificity for Alzheimer's disease

\begin{tabular}{|c|c|c|c|c|c|c|c|}
\hline Groups & No & Mean $(p g / m l)$ & $\begin{array}{l}\text { Cut off } \\
\text { (pg/ml) }\end{array}$ & $\begin{array}{l}\text { Sensitivity } \\
(\%)\end{array}$ & $\begin{array}{l}\text { Specificity } \\
(\%)\end{array}$ & Method & Study \\
\hline $\mathrm{AD}$ & 37 & $407(241)$ & & 59 & & Athena & Motter et al ${ }^{9}$ \\
\hline Healthcontrols & 20 & $212(102)$ & 312 & & 95 & & \\
\hline Other neurological disorder & 32 & $168(63)$ & & & 97 & & \\
\hline $\mathrm{AD}$ & 71 & $361(166)$ & & 39 & & Athena & Vigo Pelfrey $e t a l^{10}$ \\
\hline Neurological controls & 59 & $190(80)$ & 400 & & 100 & & \\
\hline Non-AD dementia & 25 & $235(104)$ & & & 96 & & \\
\hline Other neurological disorder & 26 & $193(127)$ & & & 88 & & \\
\hline $\mathrm{AD}$ (mildly demented) & 36 & $509(255)$ & & 81 & & Athena & Galasko et al ${ }^{19}$ \\
\hline Healthy controls & 14 & $177(82)$ & 312.00 & & NG & & \\
\hline Non-AD dementia & 9 & $166(43)$ & & & NG & & \\
\hline Other neurological disorder & 10 & $160(69)$ & & & NG & & \\
\hline $\mathrm{AD}$ & 24 & $1430(739)$ & $\sim 1300$ & 46 & & $\begin{array}{l}\text { Munroe et al } l^{15} \text { (Athena } \\
\text { antibodies) }\end{array}$ & Munroe et al ${ }^{15}$ \\
\hline Healthy controls & 14 & $816(355)$ & & & 79 & & \\
\hline Neurological controls & 26 & $790(579)$ & & & 92 & & \\
\hline Other neurological disorder & 13 & $810(441)$ & & & 85 & & \\
\hline $\mathrm{AD}$ & 27 & $10.9(4.9)$ & & 81 & & $\begin{array}{l}\text { Innogenetics (AT120 + } \\
\text { Polycl) }\end{array}$ & Vandermeeren et at \\
\hline Healthy controls & 51 & $0.1(0.55)$ & 1.1 & & 96 & & \\
\hline Other neurological disorder & 129 & $3.9(7.4)$ & & & 64 & & \\
\hline $\mathrm{AD}$ & 69 & $77.2(45.5)$ & & 99 & & $\begin{array}{l}\text { Innogenetics } \\
\text { (1st generation) }\end{array}$ & Arai $e t a l^{6}$ \\
\hline Healthy controls & 19 & $9.0(4.5)$ & 22.6 & & 100 & & \\
\hline Parkinson's disease & 7 & $15.6(7.7)$ & & & 86 & & \\
\hline ALS & 4 & $18.2(10.1)$ & & & 75 & & \\
\hline Cerebrovascular disorder & 19 & $23.4(33.1)$ & & & 84 & & \\
\hline AIDS & 4 & $49.6(37.6)$ & & & 25 & & \\
\hline Meningoencephalitis & 35 & $30.1(43.4)$ & & & $\sim 60$ & & \\
\hline Epilepsy & 6 & $14.2(10.6)$ & & & 83 & & \\
\hline Other neurological disorder & 21 & $32.8(49.2)$ & & & $\sim 67$ & & \\
\hline $\mathrm{AD}$ & 19 & $70(8)$ & NG & NG & & $\begin{array}{l}\text { Innogenetics } \\
\text { (1st generation) }\end{array}$ & Hock et $a l^{16}$ \\
\hline Neurological controls & 18 & $27(4)$ & & & NG & & \\
\hline $\mathrm{AD}$ & 23 & $279(100)$ & & NG & & Innogenetics & Tato $e t a l^{11}$ \\
\hline Neurological controls & 23 & $26(11)$ & & & NG & & \\
\hline MID (DSM-III-R) & 36 & $88(61)$ & & & NG & & \\
\hline Familial AD & 8 & $346(136)$ & & 100 & & Innogenetics (tau/g protein) & Jensen $e t a l^{8}$ \\
\hline Sporadic AD & 7 & $370(129)$ & & 95 & & & \\
\hline Neurological controls & 22 & $51(26)$ & 180 & & 93 & & \\
\hline Other neurological disorder & 31 & $155(146)$ & & & 87 & & \\
\hline $\mathrm{AD}$ & 44 & $540(280)$ & & 84 & & $\begin{array}{l}\text { Innogenetics } \\
\text { (2nd generation) }\end{array}$ & Blennow et al $l^{7}$ \\
\hline Healthy controls & 31 & $185(50)$ & 258 & & 97 & & \\
\hline VAD (NINDS-AIREN) & 17 & 445 (195) & & & 24 & & \\
\hline Frontal lobe dementia & 11 & $275(131)$ & & & 64 & & \\
\hline Parkinson's disease & 15 & $216(105)$ & & & 73 & & \\
\hline Depression & 10 & $108(13)$ & & & 100 & & \\
\hline $\mathrm{AD}$ & 11 & $254(113)$ & & NG & & $\begin{array}{l}\text { Innogenetics } \\
\text { (2nd generation) }\end{array}$ & $\begin{array}{l}\text { Skoog et } a l^{13} \text { (population based } \\
\text { study of } 85 \text { year olds) }\end{array}$ \\
\hline Non-demented & 35 & $171(78)$ & & & & & \\
\hline VAD (NINDS-AIREN) & 13 & $247(75)$ & & & NG & & \\
\hline $\mathrm{AD}$ (mildly demented) & 22 & 565 (median) & & NG & NG & $\begin{array}{l}\text { Innogenetics } \\
\text { (2nd generation) }\end{array}$ & Riemenschneider $e t a l^{17}$ \\
\hline Healthy controls & 19 & 160 (median) & NG & & NG & & \\
\hline $\mathrm{AD}$ & 16 & 325 (mean) & 225 & 88 & & $\begin{array}{l}\text { Innogenetics } \\
\text { (2nd generation) }\end{array}$ & Rosler $e t a l^{18}$ \\
\hline Neurological controls & 10 & $\sim 125$ (mean) & & & 100 & & \\
\hline Depression & 11 & $\sim 150$ (mean) & & & 91 & & \\
\hline Other neurological disorder & 20 & $\sim 200$ (mean) & & & 80 & & \\
\hline $\mathrm{AD}$ & 14 & $820(90)$ & & 100 & & Mori et $a l^{12}$ & Mori et $a l^{12}$ \\
\hline Healthy controls & 36 & $380(120)$ & $\sim 600$ & & 94 & & \\
\hline Cerebrovascular disorder & 12 & $\sim 4-500$ & & & 100 & & \\
\hline
\end{tabular}

$\mathrm{AD}=$ Alzheimer's disease; ALS = amyotrophic lateral sclerosis; AIDS = acquired immunodeficiency syndrome; MID = multi-infarct dementia; VAD = vascular dementia; $\mathrm{NG}=$ not given .

Healthy controls = people without symptoms or signs of brain disorders, including cognitive symptoms. Neurological controls = patients with minor neurological or psychiatric disorders (for example, headache, depression). Other neurological disorders = for example, multiple sclerosis, stroke, ALS, inflammatory disorders, hydrocephalus. In the 1 st generation of the innogenetics assay, a technical error in the determination of the tau standard gave rise to 6.9 times falsely low CSF-tau values.

pg/ml v n=13 with ApoE4; CSF-tau 672 (301) $\mathrm{pg} / \mathrm{ml})$. Only two MIX group patients did not possess an ApoE4 allele, making statistical analyses impossible. Neither were there any significant differences in change in CSF-tau concentrations between baseline and one year follow up, either within the Alzheimer's disease group ( $\mathrm{n}=13$ without ApoE4; change in CSF-tau -7.5 (267) $\mathrm{pg} / \mathrm{ml} \mathrm{v} \mathrm{n}=22$ with ApoE4; change in CSF-tau -3.7 (385) pg/ml), or the vascular dementia group $(n=8$ without ApoE4; change in CSF-tau $-70(121 \mathrm{pg} / \mathrm{ml} \mathrm{v}$ $\mathrm{n}=13$ with ApoE4; change in CSF-tau 23 (168 pg/ml).

\section{Discussion}

In the present paper, we confirmed previous findings ${ }^{5-19}$ of a pronounced increase in CSFtau in Alzheimer's disease. Although there were minor age differences between the diagnostic groups, we were not able to find a significant correlation between age and CSF-tau within any group. Thus the differences found are probably not caused by age differences.

We also confirmed, on a prospective community based patient sample, that CSF-tau has a high sensitivity for the diagnosis of Alzheimer's disease. In total, $41 / 43(95 \%)$ patients with Alzheimer's disease and all (100\%) patients 
with Alzheimer's disease with cerebrovascular disease (MIX) had a CSF-tau concentration above the cut off level of $306 \mathrm{pg} / \mathrm{ml}$ in healthy controls (table 1). However, the specificity was lower, as almost all (86\%) patients with vascular dementia also had high CSF-tau concentrations. Table 2 shows a summary of the literature on CSF-tau as a diagnostic marker for Alzheimer's disease. Most previous studies have found that CSF-tau has a high diagnostic sensitivity for Alzheimer's disease compared with controls, ranging from $(50-60 \%$ to $85-100 \%$, table 2 ). The sensitivity and specificity seems to mainly depend on the control population used-that is, patients with mild psychiatric/ neurological disorders $\mathrm{v}$ healthy controls (table 2). Also in the present study, the sensitivity would have been lower and the specificity higher if the NCONT group had been used to determine the cut off level.

The specificity has also been found to be relatively high compared with other dementias (for example, frontal lobe dementia), psychiatric (for example, depression), and neurological (for example, Parkinson's disease) disorders (table 2). However, when compared with vascular dementia, the specificity has been low, ${ }^{713}$ although one study found low concentrations of CSF-tau also in vascular dementia. ${ }^{11}$ In the present study, high CSF-tau concentrations were found in patients with vascular dementia, resulting in a low diagnostic specificity of CSF-tau for Alzheimer's disease. The high CSF-tau concentrations found in vascular dementia may in part be explained by the fact that, although we used strict criteria for the diagnosis, these patients may, in addition to cerebrovascular pathology, have concomitant Alzheimer's disease pathology, which is impossible to exclude clinically. Indeed, when studying another vascular dementia group in which follow up examinations including CT had been performed, an increase in CSF-tau was only found in the group without progressive leukoaraiosis. The neuropathological basis of leukoaraiosis has been suggested to be a demyelination of the white matter surrounding the cerebral ventricles, probably caused by a form of arteriolosclerosis in the long penetrating vessels supplying the white matter, although other changes, such as amyloid angiopathy ,may also contribute (for a review see Wallin and Blennow ${ }^{32}$ ). A possible theory is that patients with vascular dementia with progressive leukoaraiosis may define a more "pure" form of vascular dementia, with increasing numbers of brain infarcts as well as progressive leukoaraiosis.$^{29}$ In this group we found normal CSF-tau concentrations. By contrast, in the vascular dementia group without progressive leukoaraiosis, higher CSF-tau concentrations were found. It may be speculated whether this vascular dementia group includes patients with concomitant Alzheimer's disease pathology. Indeed, several neuropathological studies found that a high proportion $(40-80 \%)$ of clinically diagnosed patients with vascular dementia have notable concomitant Alzheimer's disease pathology. ${ }^{33}{ }^{34}$ Further studies with neuropathologically confirmed cases are needed to determine the specificity of CSF-tau as a diagnostic marker for Alzheimer's disease. However, results so far suggest that CSF-tau may help to differentiate Alzheimer's disease from several problematic differential diagnoses, such as age associated memory impairment, depressive pseudodementia, Parkinson's disease, and frontal lobe dementia.

It is as yet unclear whether the increase in CSF tau is related to the formation of PHF-tau and neurofibrillary tangles or whether it reflects the generalised neuronal and axonal degeneration found in Alzheimer's disease. After acute ischaemic stroke, there is a pronounced increase in CSF-tau within one to two weeks, which peaks after two to three weeks, and returns to normal after three to four months (Blennow et al, unpublished data). This increase probably reflects leakage of tau from damaged neurons to the CSF, supporting the view that CSF-tau reflects neuronal damage or degeneration. In Alzheimer's disease, a pronounced increase in CSF-tau is found, whereas more localised degeneration, such as in amyotrophic lateral sclerosis and Parkinson's disease, ${ }^{67}$ results in no, or minor, increases in CSF-tau concentrations, and in psychiatric disorders, such as depression ${ }^{7}$ and schizophrenia (Blennow et al, unpublished data), CSF-tau is normal. The increased release of tau into CSF as a consequence of neuronal/axonal damage is probably similar to the increased release into plasma of myocardial creatinine kinase in myocardial damage and glutamine oxaloacetic transaminase in liver tissue damage.

ApoE is involved in the pathogenesis in Alzheimer's disease, with higher frequency of the ApoE4 allele in Alzheimer's disease than in the general population. ${ }^{20-23}$ The specificity of possessing an ApoE4 allele for the diagnosis of Alzheimer's disease has been found to be high when examining neuropathologically confirmed series of demented patients. ${ }^{35-37}$ Thus patients with vascular dementia possessing an ApoE4 allele may constitute a subgroup with high prevalence of concomitant Alzheimer's disease pathology. However, CSF-tau did not differ between patients with vascular dementia with and without an ApoE4 allele.

We found it of interest to study if CSF-tau changes with time during the disease process. Most previous studies have not found any significant correlations between CSF-tau and duration of dementia, ${ }^{714}$ although Tato et $a l^{11}$ found a positive correlation between CSF-tau concentrations and duration of dementia. In the present study, CSF-tau concentrations were stable between baseline and follow up, and there was no correlation between CSF-tau and duration of dementia. These findings suggest that high CSF-tau concentrations are present during the whole course of the disease. It is possible that the same high CSF-tau concentrations are also found before the onset of clinical dementia. If so, CSF-tau may be useful for selection of patients with early memory disturbances for clinical drug trials. Studies are in progress to resolve this question. 
We also tested whether CSF-tau varies with severity of dementia, or the rate of progression of the disease. However, we were not able to find any correlation between CSF-tau and severity or rate of progression of dementia. Most previous studies have not found any significant correlations between CSF-tau and severity of disease/MMSE scores, ${ }^{6791014}$ although Tato et $a l^{11}$ found a decrease in CSF-tau with higher MMSE scores. Neuropathological studies have found that the abundance of PHF-tau related pathology correlates with the severity of dementia. ${ }^{38} 39$ If the increase in CSF-tau in Alzheimer's disease is linked to formation of PHF-tau and neurofibrillary tangles, increasing concentrations would be expected during the progression of the disease. Instead, our findings further support the notion that CSF-tau concentrations reflect the degree of neuronal degeneration, and that the activity of the degenerative process does not change during the phase of clinical dementia. However, MMSE is a relatively blunt instrument for reflecting the course of the disease, and the follow up period of one year is short. Thus further studies are needed to elucidate these issues.

Finally, higher frequency of the ApoE4 allele is found in Alzheimer's disease than in the general population. ${ }^{20-23}$ Some lines of evidence suggest a link between ApoE and tau protein. Firstly, ApoE immunoreactivity is found in neurofibrillary tangles, ${ }^{2040} 41$ which are composed of a hyperphosphorylated form of tau. ${ }^{42}{ }^{43}$ Secondly, in vitro studies have shown that ApoE3 binds to the microtubule associated protein tau with high avidity, whereas ApoE4 does not bind tau. ${ }^{24}$ ApoE3 is able to bind to each of the four microtubule binding repeats of tau, whereas ApoE4 only binds to repeat III. ${ }^{44}$ The binding between full length tau and ApoE3 is abolished by phosphorylation of tau at serine ${ }_{262}$, the only residue known to be phosphorylated on PHF-tau that lies in the microtubule binding repeat region of tau. ${ }^{44}$ However, using in situ binding techniques, ApoE has been found to bind to neurofibrillary tangles in dephosphorylated tissue, probably by phosphorylation dependent binding to tau, ${ }^{45}$ but the binding to neurofibrillary tangles did not differ between brains with different apoE alleles. ${ }^{45}$ In this study, we showed that CSF-tau concentrations did not differ between patients with Alzheimer's disease or patients with vascular dementia with and without the ApoE4 allele. These findings are in agreement with previous CSF studies, ${ }^{913} 14$ and suggest that differential binding affinity between ApoE isoforms and tau does not influence CSF-tau.

This work was supported by grants from the Swedish Medical Research Council (projects 11560 and 12103); Alzheimerfonden, Lund, Sweden; Janssen-Cilag AB, Sweden; Stiftelsen för Gamla Tjänarinnor, Stockholm, Sweden; Tore Nilssons Fond för Medicinsk Forskning, Stockholm, Sweden; Norrbottens Läns Landstings FoU Fond, Sweden; Svenska Läkaresällskapet, Stockholm, Sweden; and Åke Wibergs Stiftelse, Stockholm, Sweden. We are grateful to Mrs Christina Sjödin and Mrs Inga Volkmann for skilful technical assistance, and A Wallin and E Rehnberg for work with the control CSF material.

1 McKhann G, Drachman D, Folstein M, et al. Clinical diagnosis of Alzheimer's disease: report of the NINCDSADRDA Work Group under the auspices of Department of
Health and Human Services Task Force on Alzheimer's Disease. Neurology 1984;34:939-44.

2 Coleman PD, Flood DG. Neuron numbers and dendrite extent in normal aging and Alzheimer's disease. Neurobiol Aging 1987;8:521-45.

3 Blennow K, Bogdanovic N, Alafuzoff I, et al. Synaptic pathology in Alzheimer's disease: relation to severity of dementia, but not to senile plaques, neurofibrillary tangles, or the ApoE4 allele. 7 Neural Transm Park Dis Dement Sect 1996;103:603-18.

4 Goedert $M$. Tau protein and the neurofibrillary pathology of Alzheimer's disease. TINS 1993;16:460-5.

5 Vandermeeren M, Mercken M, Vanmechelen E, et al. Detection of proteins in normal and Alzheimer's disease cerebrospinal fluid with a sensitive sandwich enzymelinked immunosorbent assay. $f$ Neurochem 1993;61:1828linked.

6 Arai $\mathrm{H}$, Terajima M, Miura M, et al. Tau in cerebrospinal fluid: a potential diagnostic marker in Alzheimer's disease. Ann Neurol 1995;38:649-52.

7 Blennow K, Wallin A, Ågren $\mathrm{H}$, et al Tau protein in cerebrospinal fluid: a biochemical marker for axonal degeneration in Alzheimer's disease? Mol Chem Neuropathol 1995;26: 231-45.

8 Jensen $M$, Basun $\mathrm{H}$, Lannfelt L. Increased cerebrospinal fluid tau in patients with Alzheimer's disease. Neurosci Lett 1995;186:189-91.

9 Motter R, Vigo-Pelfrey C, Kholodenko D, et al. Reduction of $\beta$-amyloid peptide 42 in the cerebrospinal fluid of patients with Alzheimer's disease. Ann Neurol 1995;38: 643-8.

10 Vigo-Pelfrey C, Seubert P, Barbour R, et al. Elevation of microtubule-associated protein tau in the cerebrospinal fluid of patients with Alzheimer's disease. Neurology 1995;45:788-93.

11 Tato RE, Frank A, Hernanz A. Tau protein concentrations in cerebrospinal fluid of patients with dementia of the Alzheimer type. $\mathcal{F}$ Neurol Neurosurg Psychiatry 1995;59: 280-3.

12 Mori H, Hosoda K, Matsubara E, et al. Tau in cerebrospinal fluids: establishment of the sandwich ELISA with antibody specific to the repeat sequence in tau. Neurosci Lett 1995; 186:181-3.

13 Skoog I, Vanmechelen E, Andreasson LA, et al. A population-based study of tau protein and ubiquitin in cerebrospinal fluid in 85-year-olds: relation to dementia and cerebral atrophy, but not to the apolipoprotein E4 allele. Neurodegeneration 1995;4:433-42.

14 Nitsch RM, Rebeck GW, Deng M, et al. Cerebrospinal fluid levels of amyloid $\beta$-protein in Alzheimer's disease: inverse correlation with severity of dementia and effect of apolipoprotein E genotype. Ann Neurol 1995;37:512-8.

15 Munroe WA, Southwick PC, Chang L, et al. Tau protein in cerebrospinal fluid as an aid in the diagnosis of Alzheimer's disease. Ann Clin Lab Sci 1995;25:207-17.

16 Hock C, Golombowski S, Naser W, et al. Increased levels of tau protein in cerebrospinal fluid of patients with Alzheimer's disease - correlation with degree of cognitive impairment. Ann Neurol 1995;37:414-5.

17 Riemenschneider M, Buch K, Schmolke M, et al. Cerebrospinal protein tau is elevated in early Alzheimer's disease. Neurosci Lett 1996;212:209-11.

18 Rösler N, Wichart I, Jellinger KA. Total tau protein immunoreactivity in lumbar cerebrospinal fluid of patients with Alzheimer's disease. F Neurol Neurosurg Psychiatry 1996;60: 237-8.

19 Galasko D, Clark C, Chang L, et al. Assessment of CSF levels of tau protein in mildly demented patients with Alzheels of tau protein in mildly demented patien
imer's disease. Neurology 1997;48:632-5.

20 Corder EH, Saunders AM, Strittmatter WJ, et al. Gene dose of apolipoprotein E type 4 allele and the risk of Alzheimer's disease in late onset families. Science 1993;261:921-3.

21 Poirier J, Davignon J, Bouthillier D, et al. Apolipoprotein E polymorphism and Alzheimer's disease. Lancet 1993;342: 697-9.

22 Saunders AM, Strittmatter WJ, Schmechel D, et al. Association of apolipoprotein $\mathrm{E}$ allele 4 with late-onset familial and sporadic Alzheimer's disease. Neurology 1993;43: 1467-72.

23 Strittmatter WJ, Saunders AM, Schmechel D, et al. Apolipoprotein E: high-avidity binding to $\beta$-amyloid and
increased frequency of type 4 allele in late-onset familial Alzheimers disease. Proc Natl Acad Sci USA 1993;90:1977Alzh

24 Strittmatter WJ, Weisgraber $\mathrm{KH}$, Goedert $\mathrm{M}$, et al. Hypothesis: microtubule instability and paired helical filaments formation in the Alzheimers disease brain are related to apolipoprotein E genotype. Exp Neurol 1994;125: 163-71.

25 Han SH, Hulette C, Saunders AM, et al. Apolipoprotein E is present in hippocampal neurons without neurofibrillary tangles in Alzheimer's disease and age-matched controls. Exp Neurol 1994;128:13-26.

26 American Psychiatric Association. Diagnostic and statistical manual of mental disorders. 3rd ed, revised. Washington, DC: APA, 1987.

27 Roman GC, Tatemichi TK, Erkinjuntti T, et al. Vascular dementia: diagnostic criteria for research studies. Report of the NINDS-AIREN International Workshop. Neurology 1993;43:243-5.

28 Folstein M, Folstein S, McHugh P. "Mini-mental state" a practical method for grading the cognitive state of patients for the clinician. F Psychiatr Res 1975;12:189-98. 
29 Tarvonen-Schröoder S, Räihä I, Kurki T, et al. Clinical characteristics of rapidly progressive leuko-araiosis. Acta Neurol Scand 1995;91:399-404.

30 Kane JW, Gowland G. A method for the identification of apolipoprotein $\mathrm{E}$ isoforms employing chemical precipitation and flat bed isoelectric focusing in agarose. Ann Clin Biochem 1986;23:509-13.

31 Altman DG, Gore SM, Gardner MJ, et al. Statistical guidelines for contributors to medical journals. BMF 1983;286 1489-93.

32 Wallin A, Blennow $\mathrm{K}$. The pathogenetic basis of vascular dementia. Alzheimer Dis Assoc Disord 1991;5:91-102.

33 Jellinger KA. Diagnostic accuracy of Alzheimer's disease: a clinicopathological study. Acta Neuropathol 1996;91:219-20.

34 Kosunen O, Soininen H, Paljärvi L, et al. Diagnostic accuracy of Alzheimer's disease: a neuropathological study. Acta Neuropathol 1996;91:185-93.

35 Kakulas BA, Wilton SD, Fabian VA, et al. Apolipoprotein-E genotyping in diagnosis of Alzheimer's disease. Lancet genotyping in

36 Saunders AM, Hulette O, Welsh-Bohmer KA, et al. Specificity, sensitivity, and predictive value of apolipoprotein-E genotyping for sporadic Alzheimer's disease. Lancet 1996; 348:90-3.

37 Smith AD, Jobst KA, Johnston C, et al. Apolipoprotein-E genotyping in diagnosis of Alzheimer's disease. Lancet 1996;348:483-4.

38 Braak H, Braak E. Neuropathological stageing of Alzheimerrelated changes. Acta Neuropathol (Berl) 1991;82:239-59.
39 Arriagada PV, Growdon JH, Hedley-Whyte ET, et al. Neurofibrillary tangles but not senile plaques parallel duration and severity of Alzheimer's disease. Neurology 1992;42: 631-9.

40 Namba Y, Tomonaga M, Kawasaki H, et al. Apolipoprotein $\mathrm{E}$ immunoreactivity in cerebral amyloid deposits and neurofibrillary tangles in Alzheimer's disease and kuru plaque amyloid in Creutzfeldt-Jakob disease. Brain Res 1991;541: 163-6.

41 Wisniewski T, Frangione B. Apolipoprotein E: a pathological chaperone protein in patients with cerebral and systemic amyloid. Neurosci Lett 1992;135:235-8.

42 Grundke-Iqbal I, Iqbal K, Tung YC, et al. Abnormal phosphorylation of the microtubule-associated protein (tau) in Alzheimer cytoskeletal pathology. Proc Natl Acad Sci USA 1986;83:4913-7.

43 Ihara Y, Nukina N, Miura R, et al. Phosphorylated tau protein is integrated into paired helical filaments in Alzheimer's disease. F Biochem 1986;99:1807-10.

44 Huang DY, Weisgraber KH, Goedert M, et al. ApoE3 binding to tau tandem repeat $\mathrm{I}$ is abolished by tau serine ${ }_{262}$ phosphorylation. Neurosci Lett 1995;192:209-12.

45 Richey PL, Siedlak SL, Smith MA, et al. Apolipoprotein E interaction with the neurofibrillary tangles and senile plaques in Alzheimer disease: implications for disease pathogenesis. Biochem Biophys Res Comm 1995;208:65763. 